\title{
How certain are the reported ionic conductivities of thiophosphate-based solid electrolytes? An interlaboratory study
}

Saneyuki Ohno*a,b, Tim Bernges ${ }^{\mathrm{a}, \mathrm{b}}$, Johannes Buchheim ${ }^{\mathrm{c}}$, Marc Duchardt ${ }^{\mathrm{d}}$, Anna-Katharina

Hatz $^{\mathrm{e}, \mathrm{fg}}$, Marvin A. Kraft ${ }^{\mathrm{a}, \mathrm{b}}$, Hiram Kwak ${ }^{\mathrm{h}}$, Aggunda L. Santhosha ${ }^{\mathrm{i}}$, Zhantao Liu ${ }^{\mathrm{j}}$, Nicolò Minafra $^{\mathrm{a}, \mathrm{b}}$, Fumika Tsuji ${ }^{\mathrm{k}}$, Atsushi Sakuda ${ }^{\mathrm{k}}$, Roman Schlem ${ }^{\mathrm{a}, \mathrm{b}}$, Shan Xiong ${ }^{\mathrm{j}}$, Zhenggang Zhang, Philipp Adelhelm ${ }^{\mathrm{i}}$, Hailong Chen ${ }^{\mathrm{j}}$, Akitoshi Hayashi ${ }^{\mathrm{k}}$, Yoon Seok Jung ${ }^{\mathrm{h}}$, Bettina V. Lotsche,f,g, Bernhard Roling ${ }^{\mathrm{d}}$, Nella M. Vargas-Barbosa*e, Wolfgang G. Zeier*a,b

${ }^{a}$ Institute of Physical Chemistry, Justus-Liebig-University Giessen, Heinrich-Buff-Ring 17, D-35392 Giessen, Germany.

${ }^{b}$ Center for Materials Research (LaMa), Justus-Liebig-University Giessen, Heinrich-BuffRing 16, D-35392 Giessen, Germany.

'Institute for Technical Chemistry and Environmental Chemistry, Center for Energy and Environmental Chemistry Jena (CEEC Jena), Friedrich-Schiller-University Jena, 07743, Germany

${ }^{d}$ Department of Chemistry, Philipps-Universität Marburg, Hans-Meerwein-Straße 4, D35032 Marburg, Germany

${ }^{e}$ Max Planck Institute for Solid State Research, Heisenbergstrasse 1, 70569 Stuttgart, Germany

${ }^{f}$ Department of Chemistry, Ludwig-Maximilians-Universität (LMU) Butenandtstrasse 5-13, 81377

Munich, Germany

${ }^{g}$ Center for NanoScience, Schellingstrasse 4, 80799 Munich, Germany

${ }^{h}$ Department of Energy Engineering, Hanyang University, Seoul, 04763, South Korea

${ }^{i}$ Department of Chemistry, Humboldt-University Berlin, Brook-Taylor-Str. 2, 12489 Berlin, Germany

${ }^{j}$ The Woodruff School of Mechanical Engineering, Georgia Institute of Technology, 771 Ferst Drive, Atlanta, Georgia 30332-0245, United States

${ }^{k}$ Department of Applied Chemistry, Graduate School of Engineering, Osaka Prefecture University, 1-1

Gakuen-cho, Sakai, Osaka 599-8531, Japan

${ }^{l}$ E-conversion, Lichtenbergstrasse 4a, 85748 Garching, Germany

Owing to highly conductive solid ionic conductors, all-solid-state batteries attract significant attention as promising next-generation energy storage devices. A lot of research is invested in the search and optimization of solid electrolytes with higher ionic conductivity. However, a systematic study of an interlaboratory reproducibility of measured ionic conductivities and activation energies is missing, making the comparison of absolute values in literature challenging. In this 
viewpoint, we perform an uncertainty evaluation via a Round Robin approach using different $\mathrm{Li}$ argyrodites exhibiting orders of magnitude different ionic conductivities as reference materials. Identical samples are distributed to different research laboratories and the conductivities and activation barriers are measured by impedance spectroscopy. The results show large ranges of up to $4.5 \mathrm{mScm}^{-1}$ in the measured total ionic conductivity $\left(1.3-5.8 \mathrm{mScm}^{-1}\right.$ for the highest conducting sample, relative standard deviation $35-50 \%$ across all samples) and up to $128 \mathrm{meV}$ for the activation barriers (198 - $326 \mathrm{meV}$, relative standard deviation $5-15 \%$, across all samples), presenting the necessity of a more rigorous methodology including further collaborations within the community and multiplicate measurements.

Fast ionic conductors such as lithium and sodium thiophosphates are currently being investigated for their possible application in all-solid-state batteries. ${ }^{1,2}$ Recent research efforts have found a variety of $\mathrm{Li}^{+}$and $\mathrm{Na}^{+}$based materials such as the thiophosphates $\mathrm{Li}_{11-x} M_{1-x} \mathrm{P}_{2+x} \mathrm{~S}_{12}(M=\mathrm{Si}, \mathrm{Ge}$, $\mathrm{Sn}),{ }^{3-8} \mathrm{Li}_{2} \mathrm{~S}-\mathrm{P}_{2} \mathrm{~S}_{5}$ glasses, ${ }^{9-15} \mathrm{Li}_{6} \mathrm{PS}_{5} \mathrm{X}(\mathrm{X}=\mathrm{Cl}, \mathrm{Br}, \mathrm{I}),{ }^{16-22} \mathrm{Na}_{3} \mathrm{PS}_{4}{ }^{23-27}$ and $\mathrm{Na}_{11} \mathrm{Sn}_{2} \mathrm{PS}_{12 .}{ }^{11,28,29}$ These materials can exhibit ionic conductivities $>1 \mathrm{mScm}^{-1}$, making them viable for solid-state battery applications. Throughout the literature, however, we can find a spread in the reported ionic conductivity and activation barrier values, even within the same class of materials. Whereas significant variations in the $\mathrm{Li}^{+}$ionic transport has been reported as a function of batch variation, particle size, synthesis procedure, and even due to local sample inhomogeneity, ${ }^{30,31}$ to this day, no rigorous study on the interlaboratory reproducibility in the ionic conductivity measurement via impedance spectroscopy has been performed. Inspired by the standardization and benchmarking efforts in other communities, e.g. photovoltaics, ${ }^{32}$ in this letter we report a Round Robin study that considers the reproducibility of ionic conductivity measurements in superionic lithium thiophosphate solid electrolytes.

In this viewpoint, we chose the lithium argyrodite $\mathrm{Li}_{6} \mathrm{PS}_{5} \mathrm{X}$ as an exemplary class of materials because it provides the possibility to establish statistical trends over several orders of magnitude in the ionic conductivity, ranging from $10^{-4} \mathrm{mScm}^{-1}$ up to a few $\mathrm{mScm}^{-1}$. All test samples, namely $\mathrm{Li}_{6.6} \mathrm{P}_{0.4} \mathrm{Ge}_{0.6} \mathrm{PS}_{5} \mathrm{I}, \mathrm{Li}_{6} \mathrm{PS}_{5} \mathrm{Cl}, \mathrm{Li}_{6} \mathrm{PS}_{5} \mathrm{Br}_{0.75} \mathrm{I}_{0.25}, \mathrm{Li}_{6} \mathrm{PS}_{5} \mathrm{Br}_{0.25} \mathrm{I}_{0.75}$ and $\mathrm{Li}_{6} \mathrm{PS}_{5} \mathrm{I}$, here identified as samples (1), (2), (3), (4), and (5), respectively, were synthesized by the organizing group according to previous reports. ${ }^{18,20}$ To obtain a sufficient amount of homogeneous powder (10 g), each composition was synthesized in multiple batches, followed by X-ray diffraction of each batch to confirm the sample purities (see Figure S1). The resulting sample powders were mixed and 
homogenized, such that each participating group received $1 \mathrm{~g}$ of identical test sample for each composition. Prior to the distribution, the ionic conductivity of all homogenized samples was measured by the organizing group to make sure that the values obtained were similar to previously reported ones. All samples were supplied as powders to capture the influence of samplepreparation procedures (i.e. densification procedure, relative density, applied pressure during measurement and pellet contacting method) on the reported ionic conductivities. Each group was asked to measure temperature-dependent impedance spectra on the supplied samples, including at room temperature $\left(25^{\circ} \mathrm{C}\right)$, using the standard measuring procedure within each lab. All groups were asked to provide the measured impedance spectra and Arrhenius behaviors upon heating (see Figures S2 - S10) as well as report the calculated room temperature conductivities and the activation barriers for all samples, without providing the fitting procedure in order to avoid revealing the identity of the different groups. Further details on the sample preparation and the methodology reported of each group (labeled A to H) are given in the Supporting Information (see Table S2 - S10), which includes applied pressure upon densification (pelletizing pressure) and during measurement, contacting method, contacting material (Au sputtered and stainless steel pressed), impedance analyzer, excitation voltage, temperature and frequency range, the cell constants and employed powder masses. Hence, the statistical analysis presented here includes the measurement uncertainty of the employed impedance spectroscopy, the uncertainty in the cell constants, as well as the differences in sample preparation for the measurement itself and data analysis procedure.

Under ideal conditions, the impedance spectrum in a Nyquist representation of a polycrystalline solid electrolyte is characterized by two well-resolved semicircles and an electrode polarization. The higher frequency semicircle would then describe the ionic transport in the grains, whereas the lower-frequency semicircle describes grain boundary contributions to the ionic transport. As is typically observed for $\mathrm{Li}^{+}$conducting thiophosphates, bulk and grain boundary contributions in Li-argyrodites cannot be de-convoluted at room temperature (see Supporting Information Figures $\mathrm{S} 2-\mathrm{S} 10) .{ }^{18,20}$ Therefore, in line with the different preparation and measurement procedures, the here-reported ionic conductivity values correspond to the total ionic conductivities of all samples 
and their corresponding activation barriers. Table S11 summarizes all of the reported values used for this statistical analysis.

The obtained room-temperature conductivities are statistically analyzed using the interquartile range (IQR) method and the results are shown in Figure 1a as a box plot. Although we observe a large spread in the reported ionic conductivities for all samples (e.g. up to $4.5 \mathrm{mScm}^{-1}$ range for sample (1), we were not able to detect any outliers in any of the samples with the IQR method. In the case of samples (1) and (2), the median shows a skewness to lower and higher conductivity values, respectively, whereas for the lower conducting samples (3), (4) and (5) we observe that the mean and the median are closer to one another. Moreover, the calculated median for the lower conducting samples is close to the center of the IQR box, which suggests that the spread in the conductivity can be viewed as following a normal distribution. In the particular case of samples (1) and (2), based on this statistical analysis, the samples have very similar median total ionic conductivities, showing the difficulty in comparing different materials across laboratories.

Since we do not know what the "true" total ionic conductivity of these samples is, it is challenging to assess the best-practice procedure for the statistical analysis. We can, however, use the different measures of central tendency (median and average) as reasonable estimates for the "true" total ionic conductivity, as well as the standard deviation of the ionic conductivity as the representative expected spread in these samples for the following discussion. It needs to be mentioned that the term "true" total ionic conductivity only serves as a statistical descriptor here, it does not imply that measured values of an individual group represent a "false" ionic conductivity. Note that although the ionic conductivities of all samples range over several orders of magnitude, the calculated average values are close to the median values and high, but similar, percentages of the standard deviation are found within all samples (see Figure 1b). As the median of any dataset is less influenced by extreme values, we take it as the better estimate for the "true" total ionic conductivity, and use the calculated average to determine relative median errors for all samples (see Figure 1b). In doing so we observe that for samples with ionic conductivities $<1 \mathrm{mS} \mathrm{cm}^{-1}$ this error is less than $10 \%$, whereas in the case of the fastest conductor, the $\mathrm{Li}_{6.6} \mathrm{P}_{0.4} \mathrm{Ge}_{0.6} \mathrm{PS}_{5} \mathrm{I}$ sample, we obtain a much larger error of $22 \%$. 

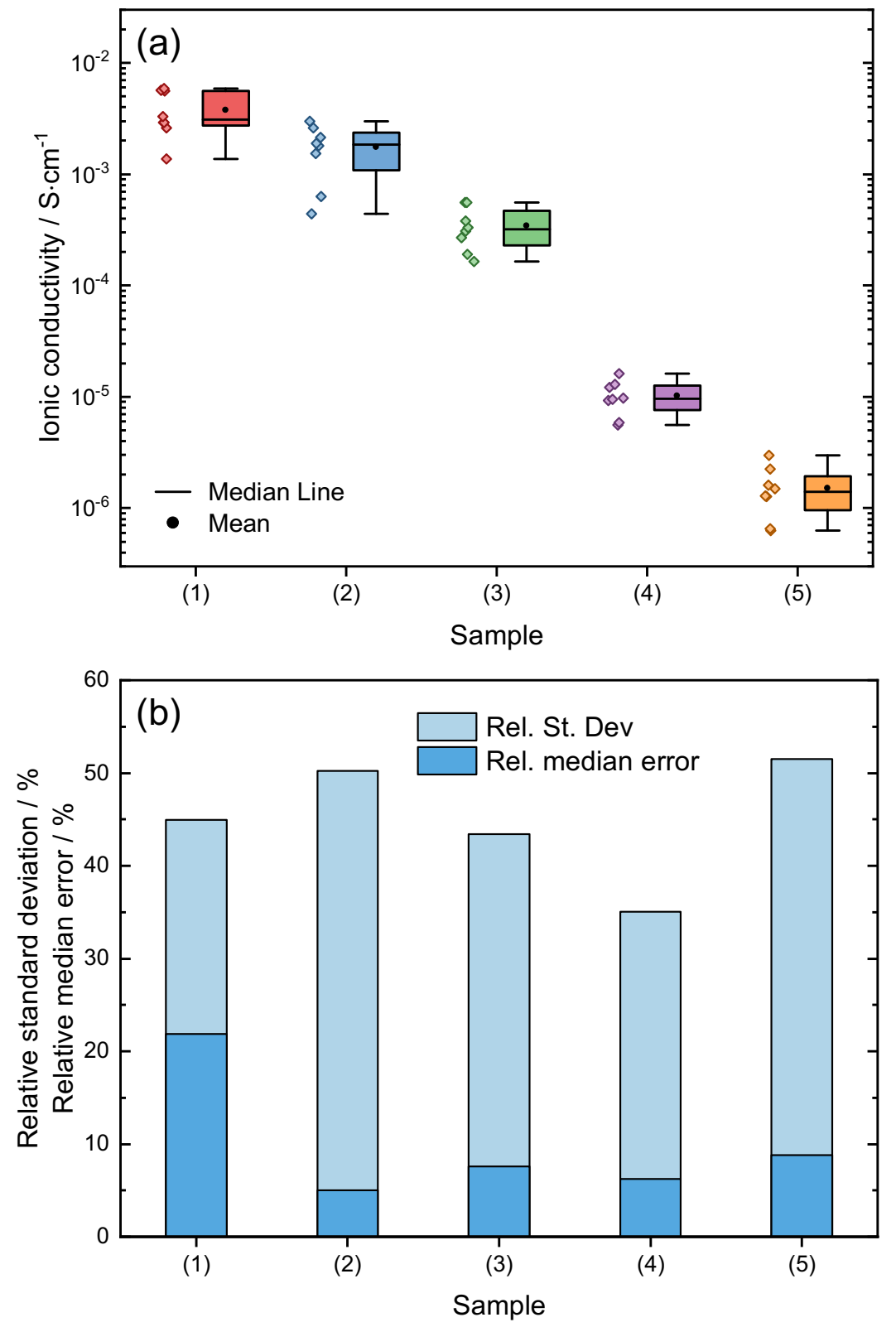

Figure 1. (a) Box plots for the room temperature total ionic conductivity for all samples in the study. (b) Percentile standard deviations and relative median error percentage for all samples. The relative median error was calculated assuming that the median represents the true total conductivity value, using $\%_{\text {error }}=\frac{\mid \text { median-average } \mid}{\text { median }} * 100$. 
In the case of the calculated activation barriers, we observe a similar spread in the reported values (see Figure 2). The IQR analysis reveals that in the case of the highest and lowest conducting samples, samples (1) and (5), respectively, one of the values with higher activation barrier is an outlier. Here, the median values for samples (3), (4), and (5) show a skewness to lower activation energies. Only in samples (1) and (2) we observe that the mean and the median are closer to one another and more centered in the IQR box, again suggesting a normal distribution of the data. In spite of the spread, the percentile standard deviations and relative median errors are much smaller than those for the ionic conductivities, likely due to the fact that the activation barrier is a parameter that is extracted from the slope of the Arrhenius plot and is therefore less sensitive to extreme values at specific temperatures. Nonetheless, even with the small deviation from the average, the values can correspond to a statistical range of up to $128 \mathrm{meV}(198-326 \mathrm{meV})$. This large range of the activation barriers is significant considering that often values of the activation barriers are reported with a large number of significant figures and changes in series of solid solutions are often within this value of the spread. Moreover, these results suggest that direct comparisons 
between experimental and theoretically calculated activation barriers are not straightforward and similar values between them does not validate neither the theory nor experimental results.
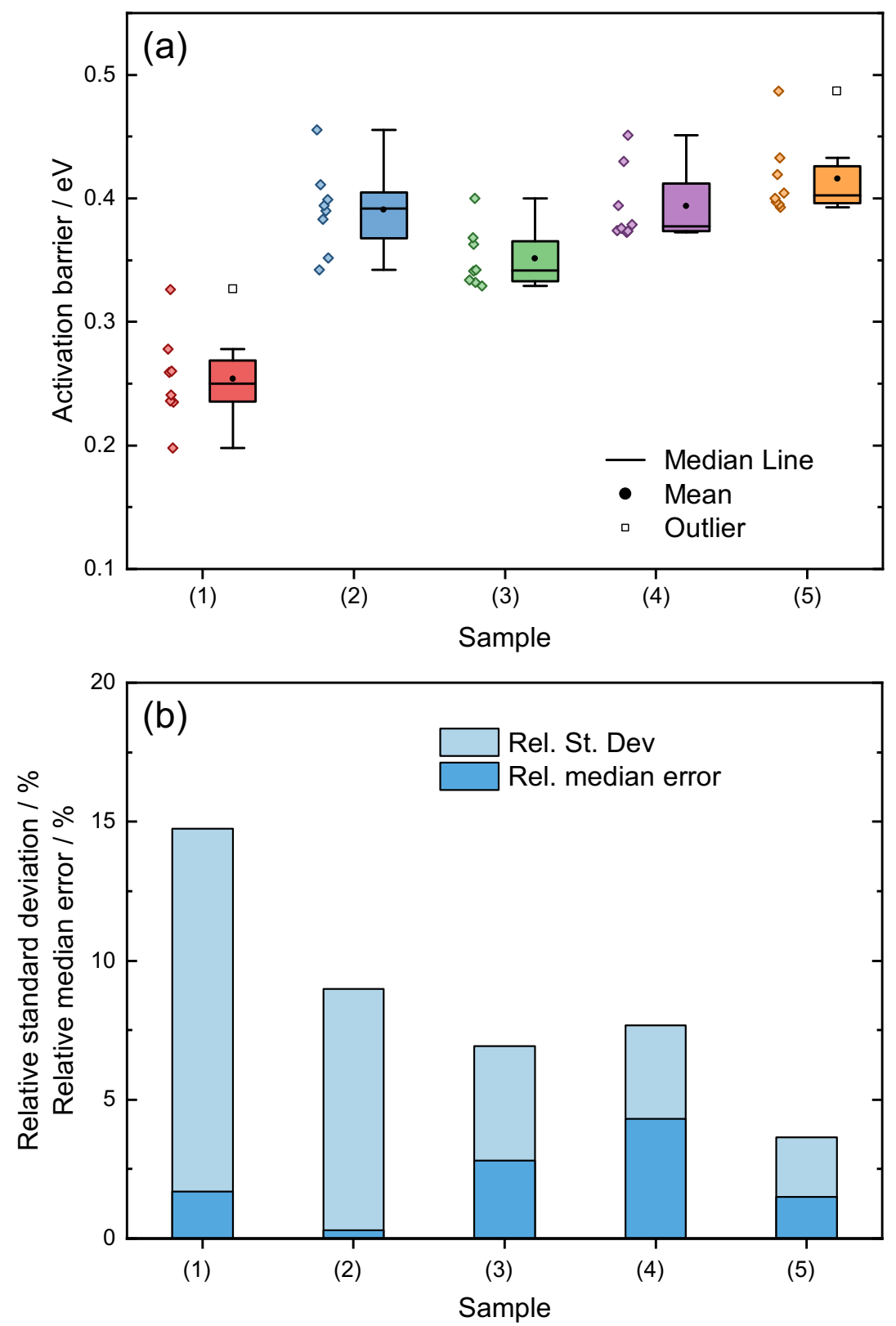

Figure 2. (a) Box plots for the activation barrier of all samples in the study. (b) Percentile standard deviations and relative median error percentage for all samples. The relative median error was calculated assuming that the median represents the true conductivity value, using $\%_{\text {error }}=$ $\frac{\mid \text { median-average } \mid}{\text { median }} * 100$. 
Since the reported conductivities correspond to total conductivities, one may expect an influence of the densification behavior or resulting relative pellet densities. While nearly $100 \%$ relative densities can be achieved in oxide ceramic-based materials via sintering, the mechanical soft nature of the thiophosphate-based electrolytes often leads to densities between $80-90 \%$ by pressing at ambient temperature. In this study a comparison of the reported ionic conductivities against the densification (pelletizing pressure) pressure, the relative pellet density, the excitation voltage, the cell constant and pellet thickness show no strong apparent trend in all samples (Figures S11-S20). As an example, Figure 3 shows the absence of clear trends for the ionic conductivity and the activation barrier of sample (2) as a function of pelletizing pressure and cell constant. Although there is no apparent trend as a function of cell constants, a qualitative trend of increasing conductivity with the relative density of the pellet can be observed. However, the trend seen as a function of relative density is not correlated to the pelletizing pressure, i.e. a higher relative density is not obtained at higher pelletizing pressures. In addition, whereas no clear influence of the contacting material was identified, an increasing conductivity with increasing applied pressure during measurement was found. This trend does not hold for the Au sputtered samples that were measured without external pressure, indicating the influence of the pressing conditions for pellet preparation on the measured ionic conductivity. These results highlight the effect of sample preparation to the microstructure of the sample and its influence on the measured conductivity. However, it should be noted that the trend with relative densities is not universally observed for 
all the samples and less prominent in lower conducting samples as seen in Figure S11 - S20, adding the difficulty of assessing the underlying mechanism of the here-observed variations.
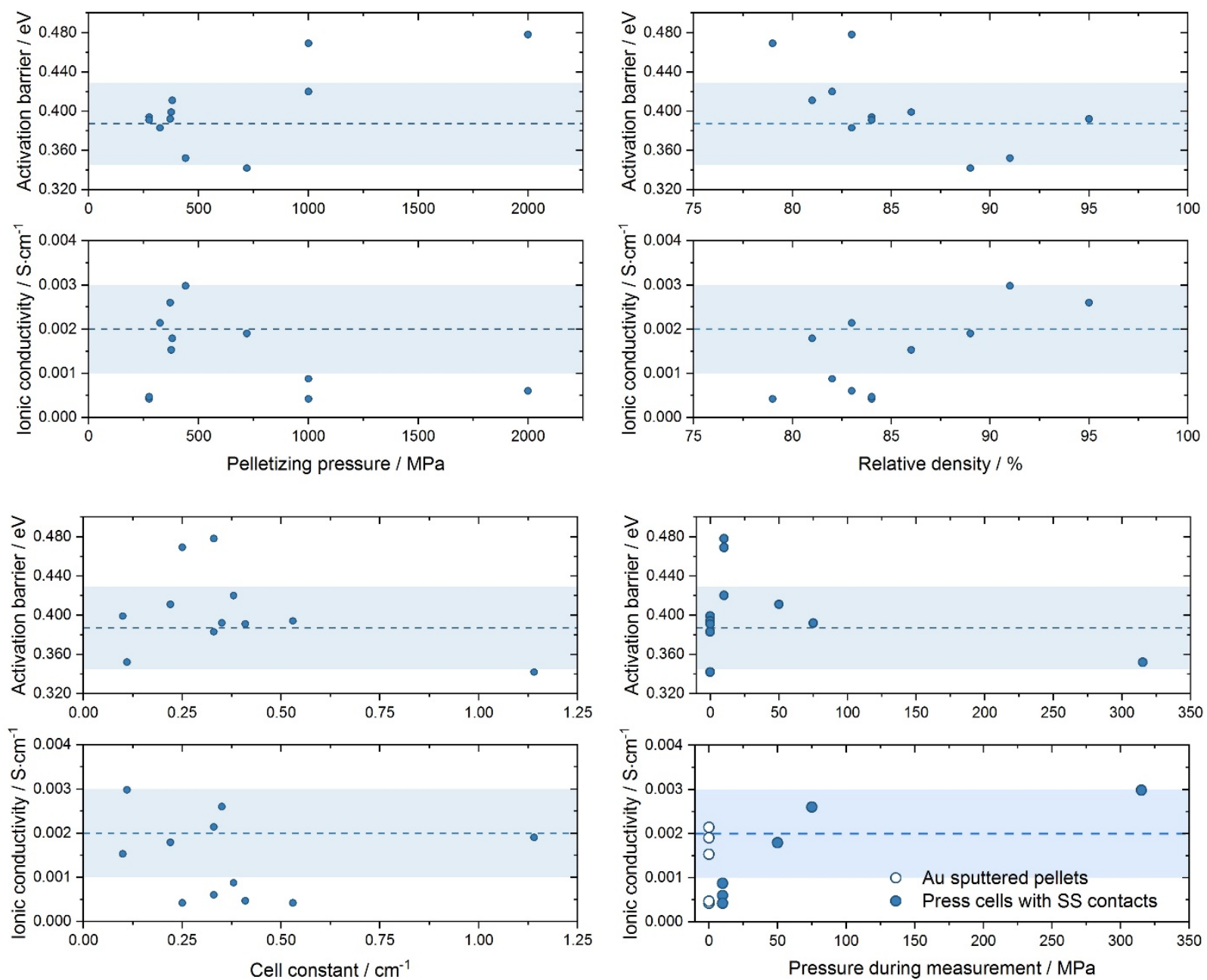

Figure 3. Spread in the total ionic conductivities and activation barriers of Sample (2) as a function of various experimental parameters.

The absence of universal trends among all the samples on one of the experimental parameters suggests a convolution of influences due to the differences in measurement setups and measurement approaches. One potential reason for the larger discrepancies at high ionic conductivities is the lack of a well-resolved semicircle. In this study, only the samples with a lower ionic conductivity show well resolved semicircles. An experimental approach for modulating the overall resistance is to change the cell constant (thickness of the pellet divided by the area of the 
electrode) and with it using enough amount of sample. Note that although changing the cell constant may help to resolve the relevant features in the impedance spectra, the cell constant itself has no influence on the intrinsic conductivity of the material, i.e. when normalizing the impedance spectra to the cell constant, the same intercept in the real impedance axis will be obtained. In other words, larger thicknesses will only change the total resistance but not the time constant and frequency range of the occurring transport processes and a deconvolution of bulk and grain boundaries may only be possible at low temperatures. ${ }^{33}$ This may be particularly important in highly conducting samples that lead to low measured resistances in which the influence of the microstructure and resulting grain resistances and microstructure may be more prominent, in comparison to the highly resistive samples. The increasing conductivity with pressure of the stainless-steel contacts, as well as the observed variation as a function of relative densities, in contrast to the values obtained by the Au sputtered samples that do not exhibit any external pressure during measurement, additionally underscores the importance of proper contacting between particles, as well as with the electrodes.

To show the influence and beneficial approach of low temperature measurements, one of the participating groups has the experimental capabilities to perform impedance measurements at temperatures down to $-140^{\circ} \mathrm{C}$. At these much lower temperatures it is often possible to separate the grain impedance from the total ionic conductivity. ${ }^{33}$ Figure S21 shows the conductivity versus inverse temperature behavior for a representative sample (2) measured to such low temperatures as well as a representative impedance spectrum of sample (2) measured at $-100^{\circ} \mathrm{C}$. At temperatures below $-75^{\circ} \mathrm{C}$, it is possible to resolve the grain conductivity contributions to the impedance spectrum. Upon fitting and extrapolating the data of grain conductivity versus inverse temperature, the extrapolated line of best fit can estimate the upper-bound values of the reported room temperature total ionic conductivities in this study. These results underscore, again, the effects of sample microstructure on the ionic conductivity, and how under the right measurement conditions it is possible to discriminate between bulk and microstructure effects.

Considering the spread in the obtained conductivities and activation barriers a few conclusions may be drawn. A larger relative median error can be clearly seen in the samples that exhibit high ionic conductivities $>1 \mathrm{mScm}^{-1}$ likely due to the convergence of different occurring processes that lead to unresolved processes, which cannot be deconvoluted without a low temperature measurement. 
Additionally, these lithium thiophosphate electrolytes are highly sensitive to atmosphere and, while structurally and chemically comparable, differences in glovebox atmospheres $\left(\mathrm{H}_{2} \mathrm{O}\right.$ and $\mathrm{O}_{2}$ content, as well as solvents present) as well as during measurement may interfere with a comparability between groups. Furthermore, pressing in these materials is needed and a certain degree of microstructural relaxation may occur after pellet consolidation and contacting, making the "sample history" a possible factor in the observed spread. Nevertheless, the large range and uncertainty in the reproducibility of the conductivity and activation barriers suggest the need to adopt a more rigorous approach in the field, as often minor improvements in activation barriers and conductivities are deemed significant.

First, it seems reasonable that for future studies showing changing conductivities and activation barriers, e.g. as a result from isovalent and aliovalent substitutions, measurements in triplicates are necessary and an accurate description of sample consolidation, contacting and measurement conditions is needed. Here, three different samples of the same composition should be measured with different cell constants (changing thicknesses as the diameter is often restricted in the cell setup), in order to change the overall cell resistance and report an average value and their respective standard deviations. Measurements at low temperatures to deconvolute processes seems crucial, ${ }^{33}$ however, this is often restricted by experimental setups. While the recommendation of triplicate measurements seems trivial, it is not often reported in literature despite the fact that these triplicates may help to more realistic uncertainties in obtained ionic conductivities and activation barriers. While the measurement of triplicates does not fully alleviate the large range that was found throughout the groups, it can provide more meaningful information on qualitative changes in the ionic transport within studies for solid solutions. In the future, measurement standards and standard materials for measurement setup validation may be needed.

Second, especially within this mechanically soft class of materials there seems to be an influence of the pressure under measurement when measured in press-cells (see Figure 3). It may hence be needed to report ionic conductivities as a function of the external pressure applied.

Third, when reporting novel electrolytes with high ionic conductivities $>1 \mathrm{mScm}^{-1}$ we suggest to send samples to a collaborative group to corroborate the obtained ionic conductivities.

Fourth, a comparison of experimental conductivity values with diffusion coefficients obtained by nuclear magnetic resonance and with-it calculations of Haven ratios may be subject to a large 
uncertainty based on the experimental range in obtained conductivities. While Haven ratios are often used to explain correlation effects, ${ }^{34}$ comparing different measurement techniques may not be very meaningful in extracting information about correlation effects in these systems.

Fifth, often theoretical calculations are internally validated by reproduction of experimental activation barriers. Despite a smaller relative median error, the larger range of up to $128 \mathrm{meV}$ in the experimentally obtained activation barriers suggests that a direct comparison between experiment and theory alone should not be used to validate theoretical results.

Overall, within the field of superionic conductors a more rigorous approach for reporting results including the experimental conditions and triplicate measurements will be needed to better understand and reliably design solid electrolytes for the use in solid-state batteries.

\section{Supporting Information}

The synthesis procedure of all samples and X-ray diffraction is shown. Further, all experimental measurement conditions of the different datasets are reported along with their statistical analysis, measured impedance spectra and Arrhenius plots as well as the comparison of the transport properties against possible sample preparation and measurement influences.

\section{AUTHOR INFORMATION}

Corresponding Authors

*saneyuki.ohno@pc.jlug.de;

*n.vargas-barbosa@fkf.mpg.de;

*wolfgang.g.zeier@pc.jlug.de;

\section{Acknowledgements}

The research was supported by the Federal Ministry of Education and Research (BMBF) within the project FESTBATT under grant numbers 03XP0117A and 03XP0177B. S.O. gratefully acknowledges the Alexander von Humboldt Foundation for financial support through a Postdoctoral Fellowship. P.A. acknowledges support within the research unit Hy-NIB (2017 FGR 
0055) funded by the ESF/Thuringia. S.X., Z.L., and H.C. acknowledge the financial support of the U.S. National Science Foundation under grant number 1706723.

\section{References}

(1) Janek, J.; Zeier, W. G. A solid future for battery development. Nat. Energy 2016, 16141.

(2) Famprikis, T.; Canepa, P.; Dawson, J. A.; Islam, M. S.; Masquelier, C. Fundamentals of inorganic solid-state electrolytes for batteries. Nat. Mater. 2019, 18, 1278-1291.

(3) Kamaya, N.; Homma, K.; Yamakawa, Y.; Hirayama, M.; Kanno, R.; Yonemura, M.; Kamiyama, T.; Kato, Y.; Hama, S.; Kawamoto, K.; et al. A lithium superionic conductor. Nat. Mater. 2011, 10, 682-686.

(4) Kato, Y.; Hori, S.; Saito, T.; Suzuki, K.; Hirayama, M.; Mitsui, A.; Yonemura, M.; Iba, H.; Kanno, R. High-power all-solid-state batteries using sulfide superionic conductors. Nat. Energy 2016, 16030.

(5) Harm, S.; Hatz, A.; Moudrakovski, I.; Eger, R.; Kuhn, A.; Hoch, C.; Lotsch, B. V. Lesson Learned from NMR: Characterization and Ionic Conductivity of LGPS-like Li7 $\mathrm{SiPS}_{8}$. Chem. Mater. 2019, 31, 1280-1288.

(6) Kuhn, A.; Koehler, J.; Lotsch, B. V. Single-crystal X - ray structure analysis of the superionic conductor $\mathrm{Li}_{10} \mathrm{GeP}_{2} \mathrm{~S}_{12}$. Phys. Chem. Chem. Phys. 2013, 15, 11620-11622.

(7) Bron, P.; Johansson, S.; Zick, K.; Schmedt auf der Günne, J.; Dehnen, S.; Roling, B. $\mathrm{Li}_{10} \mathrm{SnP}_{2} \mathrm{~S}_{12}$ : an affordable lithium superionic conductor. J. Am. Chem. Soc. 2013, 135, $15694-15697$.

(8) Krauskopf, T.; Culver, S. P.; Zeier, W. G. The bottleneck of diffusion and inductive effects in $\mathrm{Li}_{10} \mathrm{Ge}_{1-\mathrm{x}} \mathrm{Sn}_{\mathrm{x}} \mathrm{P}_{2} \mathrm{~S}_{12}$. Chem. Mater. 2018, 30, 1791-1798.

(9) Hayashi, A.; Hama, S.; Morimoto, H.; Tatsumisago, M.; Minami, T. Preparation of Li $2 \mathrm{~S}_{-}$ $\mathrm{P}_{2} \mathrm{~S}_{5}$ Amorphous Solid Electrolytes by Mechanical Milling. J. Am. Ceram. Soc. 2001, 84, $477-479$.

(10) Dietrich, C.; Weber, D.; Sedlmaier, S. J.; Indris, S.; Culver, S.; Walter, D.; Janek, J.; Zeier, W. Lithium ion conductivity in $\mathrm{Li}_{2} \mathrm{~S}_{-} \mathrm{P}_{2} \mathrm{~S}_{5}$ glasses - Building units and local structure evolution during the crystallization of the superionic conductors $\mathrm{Li}_{3} \mathrm{PS}_{4}, \mathrm{Li}_{7} \mathrm{P}_{3} \mathrm{~S}_{11}$ and $\mathrm{Li}_{4} \mathrm{P}_{2} \mathrm{~S}_{7}$. J. Mater. Chem. A 2017, 5, 18111-18119. 
(11) Hayashi, A.; Noi, K.; Sakuda, A.; Tatsumisago, M. Superionic glass-ceramic electrolytes for room-temperature rechargeable sodium batteries. Nat. Commun. 2012, 3, 856.

(12) Hayashi, A.; Ohtsubo, R.; Nagao, M.; Tatsumisago, M. Characterization of $\mathrm{Li}_{2} \mathrm{~S}-\mathrm{P}_{2} \mathrm{~S}_{5}-\mathrm{Cu}$ composite electrode for all-solid-state lithium secondary batteries. J. Mater. Sci. 2010, 45, $377-381$.

(13) Mizuno, F.; Hayashi, A.; Tadanaga, K.; Tatsumisago, M. New, highly ion-conductive crystals precipitated from $\mathrm{Li}_{2} \mathrm{~S}_{-} \mathrm{P}_{2} \mathrm{~S}_{5}$ glasses. Adv. Mater. 2005, 17, 918-921.

(14) Kato, A.; Yamamoto, M.; Sakuda, A.; Hayashi, A.; Tatsumisago, M. Mechanical Properties of $\mathrm{Li}_{2} \mathrm{~S}-\mathrm{P}_{2} \mathrm{~S}_{5}$ Glasses with Lithium Halides and Application in All-Solid-State Batteries. ACS Appl. Energy Mater. 2018, 1, 1002-1007. Oh, D. Y.; Ha, A. R.; Lee, J. E.; Jung, S. H.; Jeong, G.; Cho, W.; Kim, K. S.; Jung, Y. S. Wet-chemical tuning of $\mathrm{Li}_{3-\mathrm{x}} \mathrm{PS}_{4}(0 \leq \mathrm{x} \leq 0.3)$ enabled by dual solvents for all-solid-state lithium-ion batteries. ChemSusChem 2020, 9, 141-151.

(16) Deiseroth, H. J.; Kong, S. T.; Eckert, H.; Vannahme, J.; Reiner, C.; Zaiß, T.; Schlosser, M. $\mathrm{Li}_{6} \mathrm{PS}_{5} \mathrm{X}$ : A class of crystalline Li-rich solids with an unusually high $\mathrm{Li}^{+}$mobility. Angew. Chem. Int. Ed. 2008, 47, 755-758.

(17) Hanghofer, I.; Brinek, M.; Eisbacher, S. L.; Bitschnau, B.; Volck, M.; Hennige, V.; Hanzu, I.; Rettenwander, D.; Wilkening, M. Subsitutional disorder: Structure and ion dynamics of the argyrodites $\mathrm{Li}_{6} \mathrm{PS}_{5} \mathrm{Cl}, \mathrm{Li}_{6} \mathrm{PS}_{5} \mathrm{Br}$ and Li6PS6I. Phys. Chem. Chem. Phys. 2019, 21, 8489-8507.

(18) Kraft, M. A.; Culver, S. P.; Calderon, M.; Böcher, F.; Krauskopf, T.; Senyshyn, A.; Dietrich, C.; Zevalkink, A.; Janek, J.; Zeier, W. G. Influence of Lattice Polarizability on the Ionic Conductivity in the Lithium Superionic Argyrodites $\mathrm{Li}_{6} \mathrm{PS}_{5} \mathrm{X}(\mathrm{X}=\mathrm{Cl}, \mathrm{Br}, \mathrm{I}) . J$. Am. Chem. Soc. 2017, 139, 10909-10918.

(19) Adeli, P.; Bazak, J. D.; Park, K. H.; Kochetkov, I.; Huq, A.; Goward, G. R.; Nazar, L. F. Boosting Solid-State Diffusivity and Conductivity in Lithium Superionic Argyrodites by Halide Substitution. Angew. Chemie 2019, 58, 8681-8686.

(20) Kraft, M. A.; Ohno, S.; Zinkevich, T.; Koerver, R.; Culver, S. P.; Senyshyn, A.; Indris, S.; Morgan, B. J.; Zeier, W. G. Inducing high ionic conductivity in the lithium superionic argyrodites $\mathrm{Li}_{6+x} \mathrm{P}_{1-\mathrm{x}} \mathrm{Ge}_{\mathrm{x}} \mathrm{S}_{5} \mathrm{I}$ for all-solid-state batteries. J. Am. Chem. Soc. 2018, 140, $16330-16339$. 
(21) Ohno, S.; Helm, B.; Fuchs, T.; Dewald, G.; Kraft, M. A.; Culver, S. P.; Senyshyn, A.; Zeier, W. G. Further Evidence for Energy Landscape Flattening in the Superionic Argyrodites $\mathrm{Li}_{6+} \mathrm{P}_{1-\mathrm{x}} \mathrm{M}_{\mathrm{x}} \mathrm{S}_{5} \mathrm{I}(\mathrm{M}=\mathrm{Si}, \mathrm{Ge}, \mathrm{Sn})$. Chem. Mater. 2019, 31, 4936-4944.

(22) Yubuchi, S.; Uematsu, M.; Hotehama, C.; Sakuda, A.; Hayashi, A.; Tatsumisago, M. An argyrodite sulfide-based superionic conductor synthesized by a liquid-phase technique with tetrahydrofuran and ethanol. J. Mater. Chem. A 2019, 7, 558-566.

(23) Hibi, Y.; Tanibata, N.; Hayashi, A.; Tatsumisago, M. Preparation of sodium ion conducting $\mathrm{Na}_{3} \mathrm{PS}_{4}-\mathrm{NaI}$ glasses by a mechanochemical technique. Solid State Ionics $\mathbf{2 0 1 5}$, 270,6-9.

(24) Tanibata, N.; Deguchi, M.; Hayashi, A.; Tatsumisago, M. All-Solid-State Na/S Batteries with a $\mathrm{Na}_{3} \mathrm{PS}_{4}$ Electrolyte Operating at Room Temperature. Chem. Mater 2017, 29, 5238.

(25) Banerjee, A.; Park, K. H.; Heo, J. W.; Nam, Y. J.; Moon, C. K.; Oh, S. M.; Hong, S. T.; Jung, Y. S. Na3 $\mathrm{SbS}_{4}$ : A Solution Processable Sodium Superionic Conductor for All-SolidState Sodium-Ion Batteries. Angew. Chemie - Int. Ed. 2016, 55, 9634-9638.

(26) Moon, C. K.; Lee, H.-J.; Park, K. H.; Kwak, H.; Heo, J. W.; Choi, K.; Yang, H.; Kim, M.S.; Hong, S.-T.; Lee, J. H.; et al. Vacancy-Driven $\mathrm{Na}^{+}$Superionic Conduction in New CaDoped $\mathrm{Na}_{3} \mathrm{PS}_{4}$ for All-Solid-State Na-Ion Batteries. ACS Energy Lett. 2018, 3, 25042512.

(27) Fuchs, T.; Culver, S. P.; Till, P.; Zeier, W. G. Defect-Mediated Conductivity Enhancements in $\mathrm{Na}_{3-\mathrm{x}} \mathrm{Pn}_{1-\mathrm{x}} \mathrm{W}_{\mathrm{x}} \mathrm{S}_{4}(\mathrm{Pn}=\mathrm{P}, \mathrm{Sb})$ Using Aliovalent Substitutions. ACS Energy Lett. 2020, 5, 146-151.

(28) Zhang, Z.; Ramos, E.; Lalere, F.; Assoud, A.; Kaup, K.; Hartmann, P.; Nazar, L. $\mathrm{Na}_{11} \mathrm{Sn}_{2} \mathrm{PS}_{12}$ : A New Solid State Sodium Superionic Conductor. Energy Environ. Sci. 2017, 11, 87-93.

(29) Duchardt, M.; Ruschewitz, U.; Adams, S.; Dehnen, S.; Roling, B. Vacancy-Controlled $\mathrm{Na}+$ Superion Conduction in $\mathrm{Na}_{11} \mathrm{Sn}_{2} \mathrm{PS}_{12}$. Angew. Chem. Int. Ed. 2017, 57, 1351-1355.

(30) Smetaczek, S.; Wachter-welzl, A.; Wagner, R.; Rettenwander, D.; Amthauer, G.; Andrejs, L.; Taibl, S. Local Li-ion conductivity changes within $\mathrm{Al}$ stabilized $\mathrm{Li}_{7} \mathrm{La}_{3} \mathrm{Zr}_{2} \mathrm{O}_{12}$ and their relationship to three-dimensional variations of the bulk composition. J. Mater. Chem. A 2019, 7, 6818-6831.

(31) Kirowitz, J.; Wagner, R.; Smetaczek, S.; Brunauer, G. C.; Bonta, M.; Rettenwander, D.; 
Taibl, S.; Limbeck, A.; Amthauer, G.; Fleig, J. The origin of conductivity variations in Alstabilized $\mathrm{Li}_{7} \mathrm{La}_{3} \mathrm{Zr}_{2} \mathrm{O}_{12}$ ceramics. Solid State Ionics 2018, 319, 203-208.

(32) Gevorgyan, S. A.; Zubillaga, O.; María, J.; Seoane, V. De; Machado, M.; Alturk, E.; Tore, N.; Voroshazi, E.; Aernouts, T.; Müllejans, H.; et al. Round robin performance testing of organic photovoltaic devices. Renew. Energy 2014, 63, 376-387.

(33) Bron, P.; Dehnen, S.; Roling, B. Li ${ }_{10} \mathrm{Si}_{0.3} \mathrm{Sn}_{0.7} \mathrm{P}_{2} \mathrm{~S}_{12}$ - A low-cost and low-grain-buondary resistance lithium superionic conductor. J. Power Sources 2016, 329, 530-535.

(34) Vargas-Barbosa, N. M.; Roling, B. Dynamic ion correlations in solid and liquid electrolytes: how do they affect charge and mass transport. ChemElectroChem 2020, 7, $367-385$. 